\title{
The Texture Animator
}

\author{
Damjan Strnad and Nikola Guid \\ Faculty of Electrical Engineering and Computer Science, University of Maribor, Slovenia
}

\begin{abstract}
This paper discusses three distinct techniques for animation of procedural textures and describes the assisting software tool. Animation is attained by moving the rendered point before texture evaluation, changing the definition of texture space or changing the texture colour mapping. Examples are given for textures that base on noise and turbulence functions in order to simulate natural phenomena. Some phases of texture animation process can be automated by using the code generator supported by a library of animation effects. Aspects of practical implementation are discussed and Renderman compliant code is presented.
\end{abstract}

Keywords: texture animation, procedural texture, code generator, Renderman specification

\section{Introduction}

Texturing is one of fundamental procedures in modern computer graphics. It significantly increases image realism and is essential for achieving the so-called photo-realism in artificially constructed and rendered scenes. Textures may be derived by scanning a photo or they can be procedurally defined. Procedural textures are today's topic. They can be defined over arbitrary number of dimensions, although 3D solid textures get most practical use today. Procedural texture value is determined by program code and interpretation of that value depends on the renderer. The most common use of textures is surface colour representation that will also be adopted in this article.

Still images of textures have very limited usage, especially if the texture represents inherently unsteady phenomenon (e.g. fire, water, ...). Animated sequences consist of several still images (frames) that differ in object and light source positions, viewpoints and object geometries. To change object's appearance, we might also use slightly different texture for each frame. However, it is desired that the texturing function takes care of temporal variance of its output. We call such mutable texture an animated texture.

To animate a texture we need to incorporate the temporal component into the texturing function. This is usually done in sense of a new input parameter, representing frame number or some other aspect of animation progress. We prefer to use progress indicator with values normalized to the range $[0,1]$ and call it the time parameter.

The integration of new temporal dimension into existing static texture should be done in a way that provides the animated texture with following properties:

- it changes rather smoothly over time,

- its temporal development is sensible for the simulated phenomenon and avoids periodicity and

- the computational overhead is small compared to the static version of a texture.

All of examples given in this paper are described using Renderman shading language specification that is widely used among experts in photorealistic image synthesis (Pixar, 2000). It allows us to write user-defined procedural texture functions, called shaders. Many types of shaders exist that can be attached to any object in scene, but we are primarily concerned with surface shaders.

Any user-defined shader is invoked many times during the rendering of an object it is attached to. It must provide sample point's colour and opacity. Shader environment is established during each invocation, defining a set of input variables like position of a rendered surface point, surface normal at that point, camera position, 
viewing direction etc. Surface shader can use that information to determine the texture value and set output colour and opacity variables. The colour and opacity returned by the shader are actually used to paint the object, so ambient, diffuse, specular or any other lighting must be accounted for inside the shader procedure. Besides environment variables, each shader can have input parameters.

Our aim is to present approaches to writing animated shaders that accept normalized time information in one of parameters. Renderman shading language will be our tool to express the implementation with practical background.

We shall describe three distinct approaches for writing animated Renderman shaders. The order in which they will be discussed corresponds to the order in which changes are made to the shader procedure.

\section{Animation techniques and their automation}

The three approaches of our discussion are:

- time-based moving of the point before texture evaluation,

- altering of the texture space over time and

- altering of the colour mapping function over time.

Advantage of the first two approaches over the third one is that they can be used for various kinds of textures, not only those representing the surface colour, since bump and displacement textures usually don't use an extra mapping from texture to feature space.

As we shall see, animating arbitrary textures can be automated to some extent, depending on the technique used. We have therefore written a relatively simple animating code generator (we call it the texture animator) that can save us a lot of tedious work. It is supported by a growing database of animating effects that are additionally modulated through clear user interface. The code produced is Renderman shading language compliant so it can be included in any shader relatively independently (minor variable renaming may be necessary).

\subsection{Moving the rendered point}

A very powerful technique for texture animation is to move the rendered point along some path before evaluating it by the texture function. Texture value surely depends on point's location so by changing the latter, texture value changes as well. Animation is attained by using the time component to decide on the length of the move along the path. The visual effect reflects the point movement but in opposite direction (e.g. if we move points to the left, the texture moves to the right). Linear paths are extremely simple but also quite useless. Much more interesting are helical paths (Ebert et al., 1998), spiral (vortex) paths and cycloidal paths.

Moving the point along some path can be easily automated. At the moment our texture animator includes most frequently used paths.

There is one problem with previously mentioned paths - they are too regular to be natural. Smoke rising is rarely as perfect as a smooth helical path, although it manifests such behaviour at a large scale. Small-scale deviations are inserted by means of a turbulent distortion that enhances animation veracity a great deal.

A sample output of texture animator with perturbed cycloidal path is:

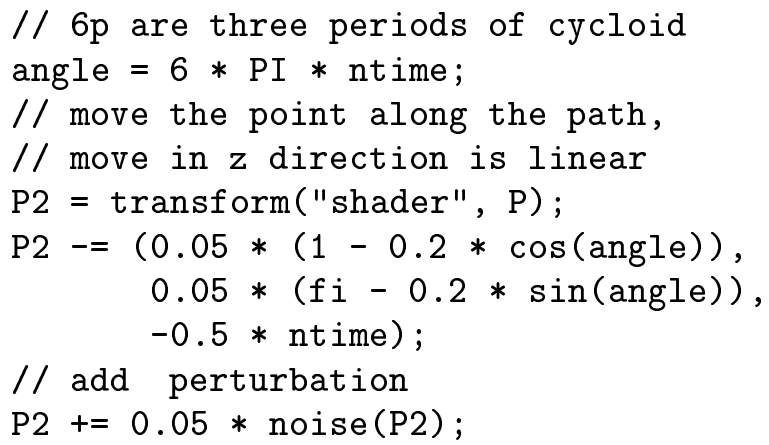

An exemplary image sequence of animated flame texture is shown in fig. 1 . The animation path is a vertical cycloid. Frames follow from top left to bottom right in rows.

Further improvements can be made since natural phenomena very rarely develop so onedirectionally. Many directions of texture movement can be achieved using virtual objects that work as attractors to the texture points. The 


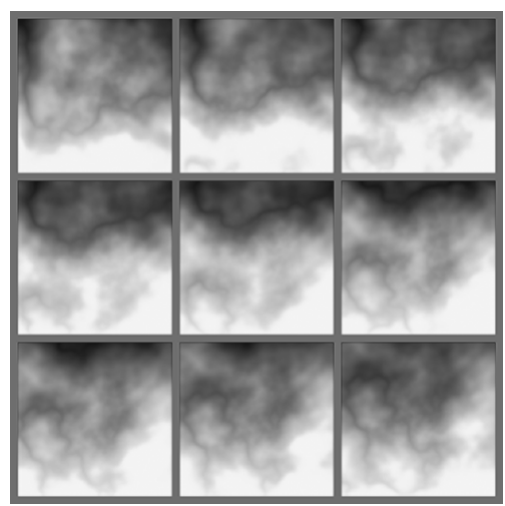

Fig. 1. Animating flame by moving rendered points along cycloidal path.

shape and strength of attractor can vary over time, producing very practical animations. Again, spherical and linear attractors are easiest to do, while possibilities are almost unlimited (Ebert et al., 1994). Repulsors are negative attractors.

We have extended the texture animator to include few primitive attractors/repulsors. Each attractor is described by several characteristic parameters, such as centre point and radius for a spherical attractor. Each rendered point is moved in direction away from the closest point on the attractor, so that the visual effect is opposite. The length of the move depends on the attractor strength and point-attractor distance.

Fig. 2 is a sequence of frames showing the wideangle objective effect of a strengthening circular attractor/repulsor combination produced by the following chunk of automatically generated code:

// circular attractor/repulsor is

// described with its centre point

// and radius

away = P-centre;

1 = length (away);

$/ /$ if inside the circle then

// elongate the move

if ( $1<$ radius) away $*=2$;

// move the point

P2 = transform ("shader", P);

P2 += ntime*away;

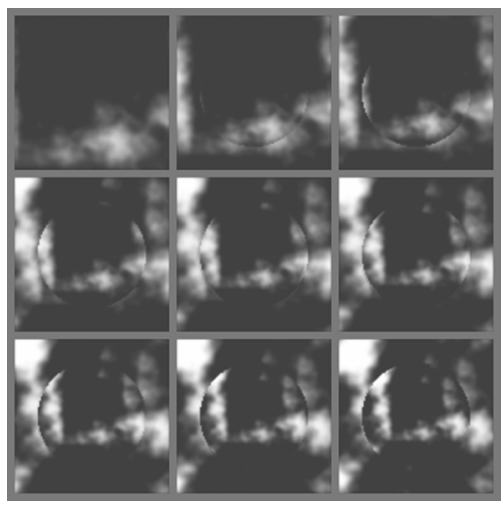

Fig. 2. Spherical attractor producing wide-angle objective effect.

\subsection{Changing the texture space}

Changing the texture space is probably the most natural way of texture animation. It requires direct modification of texture function core and thus necessitates its detailed knowledge. Texture value is seldom calculated in one step, but rather gained by combining several quantities. Some of those quantities are given in parameter values and some are calculated separately. In the end, independent quantities $q_{i}$ may be simply added together to give the final texture value $T$ :

$$
T=\sum_{i} w_{i} q_{i}
$$

Values $w_{i}$ are user-defined weights. Normalized time component $t$ can be comprised in calculation using any function $f(t)$ that maps range $[0,1]$ onto itself. Simplest cases of $f$ are $f(t)=t$ and $f(t)=1-t$. Texture evaluation then sounds:

$$
T=\sum_{i} w_{i} f_{i}(t) q_{i}
$$

Continuous mapping provided by functions $f_{i}$ assures smooth texture animation.

Unfortunately, texture value is hardly ever produced by a simple weighted summation. Participating quantities are usually interdependent. The extreme case would be if the amount of every quantity would influence the amount of next quantity, so the texture value would be the given by the following equation:

$$
T=g_{n}\left(g _ { n - 1 } \left(\ldots\left(g_{1}\left(q_{0}\right) \ldots\right)\right.\right.
$$


Quantity $q_{0}$ represents the input information. Similar approach as before can be taken to animate such texture. Amount of any intermediate quantity can be regulated by the time component in the following way:

$$
T=g_{n}\left(g _ { n - 1 } \left(\ldots \left(f ( t ) g _ { i } \left(\ldots\left(g_{1}\left(q_{0}\right) \ldots\right)\right.\right.\right.\right.
$$

Usually the truth is somewhere between the two presented extremes. Useful textures are composed of several independent quantities that are calculated in more than one step, comprehending various offsets, distortions and the like.

Practical drawbacks of texture space animation are that it is not very intuitive and is also hard to automate, so texture animator is primarily used to insert typical cases of $f$ functions.

To demonstrate the richness and power of this technique, we have used a simplified version of fractal terrain generator (Musgrave et al., 1989). Fig. 3 shows the image sequence of a developing planet surface. Two quantities are being regulated:

- the amplitude multiplier in the fractal loop is linearly increased, causing the coastline to reshape and islands to appear and

- the terrain altitude is evened out by a quadratic time function, producing the effect of vegetation extinction.

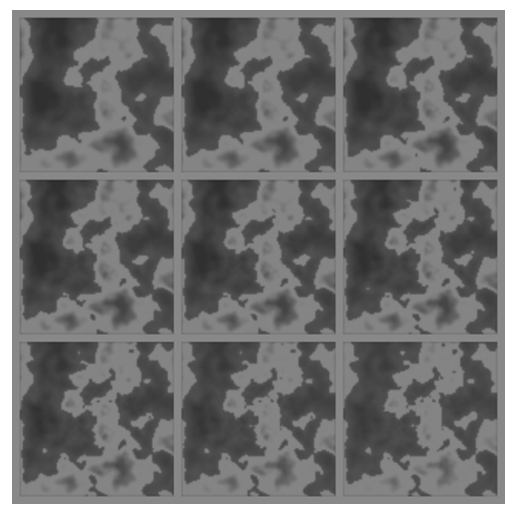

Fig. 3. Development of planet texture with time-dependent texture function.

The relevant code reads:

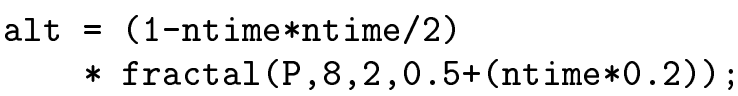

\subsection{Changing the colour mapping}

Once the texture value for a given point is determined, it can be immediately used as a displacement or bump map (we keep in mind that texture may be vector-valued). In that case no additional mapping is required. With colour textures, however, the common practice is to transform the scalar texture value to the vector colour representation (mostly RGB).

In the continuation, we shall assume that the colour mapping function transforms normalized texture values into colours using colour palette. Colour palette may contain arbitrary number of colours. For example, if colour palette contains 11 colours, then texture value 0.0 maps to the first colour, texture value 0.1 maps to second colour and so on. Texture value 1.0 maps to the last colour in the palette. Intermediate texture values map to colours that are interpolation of two corresponding colours from the palette.

Such notion of colour mapping is in practice prevalent with mapping function being the interpolating curve, usually some sort of cubic colour spline (Musgrave, 1991). In spline terminology the colours in the palette are called knots. Spline-based colour mapping is particularly common in Renderman compliant shaders because the shading language directly supports cubic Catmull-Rom colour splines via built-in function called spline.

Two different techniques can then be used to animate the texture:

- changing the colour palette and

- changing the interpolating function.

If we are bound to use colour splines, the only way to animate the colour mapping is to temporally change knot values. This corresponds to the first technique listed above. Implementation includes at least two colour palettes - starting colour palette for the first frame and ending colour palette for the last frame. Intermediate colour palettes are gathered by linear interpolation. Higher-order interpolation and userdefined weights can also be used for special applicability. If $C_{i}^{0}$ and $C_{i}^{1}$ are the $i$-th colours of the starting and ending colour palettes, respectively, then the $i$-th colour of the active colour palette with respect to the normalized animation 


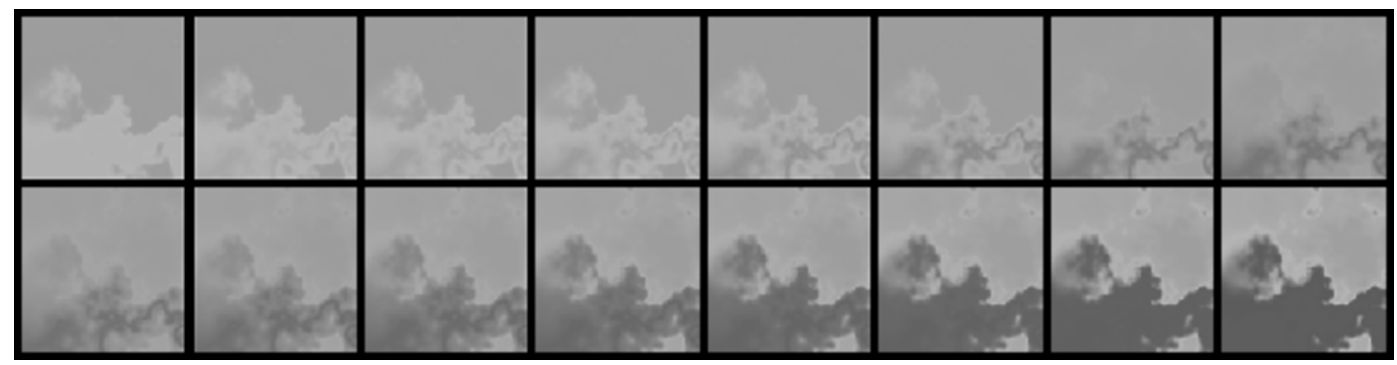

Fig. 4. Process of colour blending with time dependent colour palette.

time $t$ is in case of linear palette interpolation given by:

$$
C_{i}^{t}=\left(1-w_{i} f_{i}(t)\right) C_{i}^{0}+w_{i} f_{i}(t) C_{i}^{1}
$$

Weights $w_{i}$ must all lie in the range $[0,1]$ and functions $f_{i}$ must be bijective on that range. All power functions meet that condition.

Three or more colour palettes and another spline interpolation between them can supply additional animating power. We emphasize that two interpolations are taking place here: interpolation of colour palettes and interpolation between colours in each palette.

Implementation of such scheme using the shading language is very elegant:

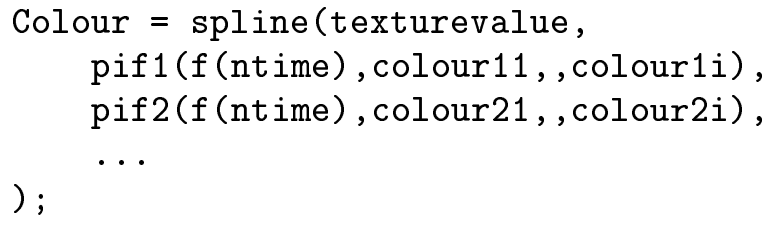

The outer spline function does the interpolation between colours in the palette. The latter are determined by inner interpolation functions, denoted by pif $x$ above. Depending on what kind of interpolation exists between colour palettes, functions mix, smoothstep or spline would be used in place of pif $x$ for linear, cubic or general spline interpolation, respectively.

Second listed option is available with parametershapable interpolators. The simplest example is the Hermite spline that can be shaped by changing tangent vectors in all points. Many variants of B-splines exist that can also be parametrically fine-tuned. Any such interpolating function can be used as long as it is reshaped in order to return colour values.
We have extended texture animator with colour mapping support routines. Up to four colour palettes may be constructed by visual picking up of colours and the desired interpolation scheme is built.

Fig. 4 shows phases of colour absorption process. The animation may not be extremely useful in this form but is typical for the technique discussed here. The only thing changing is the colour palette of 10 colours. Fig. 5 shows apple ripening, attained by linear interpolation between colour palettes of four colours only. Richer collection of effects can be achieved with combinations of all mentioned techniques.

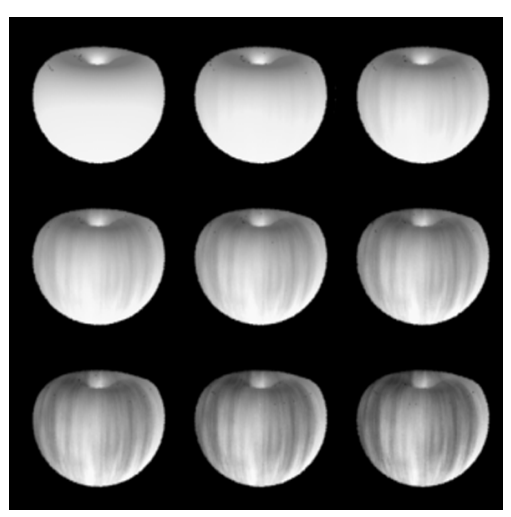

Fig. 5. Apple ripening with time dependent colour palette.

\section{Conclusion}

Texture animation has already proven its importance in practice, but not all aspects of it have been presented here. Hypertextures (Perlin and Hoffert, 1989) and other textures that are based on volume density functions and require volume rendering are yet to be extensively animated. The same is true for many explicit procedural textures like reaction-diffusion texture (Turk, 
1991). Approaches discussed here are also applicable to some of these areas. They are by no means all the choice we have. Other possibilities include procedural textures that evaluate the rendered point in world space, so that any object transformation reflects in texture change.

Automation of some of the techniques has proven to be very helpful, while texture space animation remains mainly manually driven. That problem is common to texture synthesis and animation and should be challenged by expert texture generation tools and specialized interfaces (Worley, 1993).

\section{References}

[1] D. S. Ebert, W. Carlson, R. E. Parent, Solid Spaces and Inverse Particle Systems for Controlling the Animation of Gases and Fluids. The Visual Computer, 10 (1994), 179-190.

[2] D. S. Ebert, F. K. Musgrave, D. Peachey, K. Perlin, S. Worley, Texturing and Modeling $-a$ Procedural Approach, Academic Press, San Diego, 1998.

[3] F. K. Musgrave, C. E. Kolb, R. S. Mace, The Synthesis and Rendering of Eroded Fractal Terrains, SIGGRAPH '89 Proceedings, 23 (1989), $41-50$.

[4] F. K. Musgrave, A Random Color Map Animation Algorithm, in Graphics Gems II, (1991) pp. 134-137, Academic Press, Boston.

[5] K. Perlin, E. M. Hoffert, Hypertexture, SIGGRAPH '89 Proceedings, 23 (1989), 253-262.

[6] PIXAR, The Renderman Interface: Specification 3.2, Pixar, San Rafael, 2000.

[7] G. TURK, Reaction Diffusion Textures, Computer Graphics, 25 (1991), 289-298.

[8] S. WORLEY, Practical Texture Implementation, Procedural Modeling and Rendering Techniques course notes, ACM SIGGRAPH 1993, 12.

Received: October, 2000 Accepted: November, 2000

Contact address:

Damjan Strnad

Laboratory for Computer Graphics and Artificial Intelligence

Department of Computer Science

Faculty of Electrical Engineering and Computer Science

University of Maribor

Smetanova 17

SI-2000 Maribor, Slovenia

phone: $++38662220-7472$

e-mail: damjan strnadQuni-mb
DAMJAN STRNAD is an Assistant at the University of Maribor and member of a Laboratory for Computer Graphics and Artificial Intelligence. His research interests include computer graphics and geometric modelling, specifically photo-realistic rendering and texturing. Strnad received a B.Sc. and M.Sc. in computer science from the University of Maribor, Slovenia in 1998 and 2000, respectively. He is a member of Eurographics.

NIKOLA GUID is presently a Full Professor at the Faculty of Electrical Engineering and Computer Science in the Univeristy of Maribor, Slovenia, and a head of Laboratory for Computer Graphics and Artificial Intelligence. His current research interests are computer graphics, computer aided geometric design, geometric modelling, computer simulation, search strategies, and knowledge representation. He wrote two textbooks on computer graphics. Guid received both a B.Sc. and M.Sc. in electrical engineering from the University of Ljubljana in 1974 and 1977, respectively, while a Ph.D. from the University of Maribor in 1984. He is a member of the IEEE and Eurographics. 